\title{
Inflammatory Diseases of the Meninges
}

\author{
Stefan Rohde
}

\section{CONTENTS}

11.1 Introduction 169

11.2 General Pathophysiological Considerations 170

11.3 Bacterial Meningitis 172

11.3.1 Epidemiology, Clinical Presentation, Therapy 172

11.3.2 Imaging 172

11.3.3 Differential Diagnosis 177

11.4 Tuberculous Meningitis 177

11.4.1 Epidemiology, Clinical Presentation, Therapy 177

11.4.2 Imaging 178

11.4.3 Differential Diagnosis 178

11.5 Viral Meningitis 178

11.5.1 Epidemiology, Clinical Presentation, Therapy 178

11.5.2 Imaging 180

11.5.3 Differential Diagnosis 181

11.6 Fungal Meningitis 181

11.6.1 Epidemiology, Clinical Presentation, Therapy 181

11.6.2 Imaging 181

11.6.3 Differential Diagnosis 181

11.7 Neurosyphilis 182

11.7.1 Epidemiology, Clinical Presentation, Therapy 182

11.7.2 Imaging 182

11.7.3 Differential Diagnosis 182

11.8 Neurosarcoidosis 182

11.8.1 Epidemiology, Clinical Presentation, Therapy 182

11.8.2 Imaging 182

11.8.3 Differential Diagnosis 183

References 183

\section{S. ROHDE, MD}

Division of Neuroradiology, Heidelberg University Medical Center, Im Neuenheimer Feld 400, 69120 Heidelberg, Germany

\section{S U M M A R Y}

Neuroimaging is of major importance in all cases of suspected infectious meningitis in order to get quick information about the extent of the disease, typical lesion patterns, and potential complications, such as hydrocephalus, involvement of the underlying brain parenchyma, or vasculitis. In bacterial meningitis, abnormal and asymmetrical enhancement of the leptomeninges and the subarachnoid space is typical. Initial neuroimaging has to rule out infectious foci of the skull base such as purulent sinusitis or mastoiditis. In patients with focal deficits or seizures, MRI is the tool of choice to diagnose vascular or septic complications. Neoplastic, viral, or fungal infections of the CNS may present with similar changes of the meninges; however, fungal meningitis normally causes a thicker and more nodular enhancement. In case of basal accentuation of the leptomeningeal contrast enhancement and conspicuous signal changes in the basal cisterns, one has to consider tuberculous meningitis for differential diagnosis, especially in patients with HIV infection. Non-infectious causes of meningeal enhancement comprise several primary and secondary tumors (e.g., CNS lymphoma, medulloblastoma, or breast cancer), granulomatous diseases, and post-operative changes.

\section{1 \\ Introduction}

Inflammation of the protective membranes covering the brain and spinal cord, known collectively as the meninges, may develop in response to a number of causes, most prominently bacteria and viruses, but also fungi, 
Table 11.1. Probable causes of predominant leptomeningeal contrast enhancement in neuroimaging

\begin{tabular}{|lll|}
\hline Etiology & Type of enhancement & Remarks \\
\hline Bacterial meningitis & $\begin{array}{l}\text { Asymmetrical, thin, linear enhancement } \\
\text { of the leptomeninges and the SAS, typically } \\
\text { affects convexities }\end{array}$ & $\begin{array}{l}\text { Tuberculous meningitis predominantly } \\
\text { affects the basal meninges and cisterns, often } \\
\text { cranial nerve involvement }\end{array}$ \\
\hline Viral meningitis & Thin, linear enhancement & Often no enhancement \\
\hline Fungal meningitis & Thick, lumpy, or nodular enhancement & $\begin{array}{l}\text { May also cause pachymeningeal enhance- } \\
\text { ment }\end{array}$ \\
\hline $\begin{array}{l}\text { Neoplastic } \\
\text { ("carcinomatous") meningitis }\end{array}$ & $\begin{array}{l}\text { Wide range from thin and linear to thick, } \\
\text { lumpy, or nodular enhancement; may also } \\
\text { involve dural structures }\end{array}$ & $\begin{array}{l}\text { May also involve underlying cortex or pa- } \\
\text { chymeningeal structures; most often in CNS } \\
\text { lymphoma, breast cancer, medulloblastoma, } \\
\text { and ependymoma }\end{array}$ \\
\hline Neurosarcoidosis & $\begin{array}{l}\text { Wide spectrum of leptomeningeal } \\
\text { (and pachymeningeal) enhancement, } \\
\text { especially in basal cisterns, often cranial } \\
\text { nerve involvement }\end{array}$ & - \\
\hline
\end{tabular}

parasites, radiation, or neoplasm. Moreover, a number of systemic diseases, such as sarcoidosis or vasculitis, may involve the meninges.

In cases of acute infectious meningitis, rapid diagnosis and identification of the probable pathogen is mandatory, as early effective antimicrobial treatment is of major importance. While examination of the CSF, laboratory analysis, and biopsy remain the gold standard to identify the infectious agent, neuroimaging plays an important role in visualizing typical lesion patterns, the extent of the disease, and the potential involvement of deeper brain structures. In addition, imaging may help to identify sources of infection, such as fractures of the skull base, infection of the temporal bone or mastoid, and to rule out other, non-infectious causes of inflammatory disease of the meninges, such as granulomatous or neoplastic processes.

\section{2}

\section{General Pathophysiological Considerations}

Anatomically, the meninges consist of three layers: the dura mater; the arachnoid mater; and the pia mater. However, for a better understanding of meningeal inflammation in neuroimaging, it is helpful to use the anatomic-functional classification of lepto- and pachymeninges (SMIRNiotopoulos et al. 2007).

The leptomeninges (skinny meninges) comprise the membranes of the arachnoidea and the pia mater; the latter one follows the surface of the brain. The space between the arachnoid mater and the pia mater is the subarachnoid space (SAS). The vessels within the leptomeninges have a blood-brain barrier (BBB) that inhibits leakage of endogenous and exogenous serum compounds under physiological conditions. Infections of the leptomeninges and the SAS cause multiple pathophysiological changes in brain homeostasis. The SAS is infiltrated with inflammatory cells, which release glycoproteins and toxins that affect the tight junctions of the vascular endothelium, leading to a breakdown of the BBB. Serum fluid, proteins, and cells leak into the SAS and the adjacent cortex and cause interstitial and parenchymal edema. The breakdown of the BBB also allows contrast material to leak from the vessels into the CSF. The typical pattern of leptomeningeal enhancement follows the surface of the brain and fills the SAS of the sulci and cisterns. While bacterial and viral meningitis are supposed to exhibit enhancement that is thin and linear, fungal meningitis and neoplastic infiltration of the leptomeninges may produce thicker, lumpy, or nodular enhancement in the SAS. A list of the potential causes of leptomeningeal enhancement is given in Table 11.1.

The pachymeninges (thick meninges) consist of the dura mater and the outer layer of the arachnoid mater; they follow the dural flections of the inner table of the skull, the falx cerebri, tentorium cerebelli, and the venous sinus. The vessels within the dura mater do not produce a BBB; thus, serum compounds, such as albumin, fibrinogen, or contrast agent, can leak into the dura mater. Therefore, the pachymeninges can be seen 
Table 11.2. Probable causes of predominant pachymeningeal contrast enhancement in neuroimaging

\begin{tabular}{lll}
\hline Etiology & Type of enhancement & Remarks \\
\hline $\begin{array}{l}\text { Intracranial hypotension, e.g., } \\
\text { after surgery, lumbar puncture }\end{array}$ & $\begin{array}{l}\text { Linear enhancement of the dura and falx } \\
\text { cerebri with global homogeneous thickening }\end{array}$ & $\begin{array}{l}\text { Additional findings: enlargement of the } \\
\text { pituitary gland; low cerebellar tonsils; } \\
\text { subdural effusions / hemorrhage }\end{array}$ \\
\hline Meningioma & $\begin{array}{l}\text { Curvilinear enhancement adjacent to the } \\
\text { bulky tumor ("dural tail") }\end{array}$ & - \\
\hline Metastases & Thick, nodular irregular enhancement & $\begin{array}{l}\text { Most often from breast cancer and prostatic } \\
\text { cancer }\end{array}$ \\
\hline $\begin{array}{l}\text { Granulomatous diseases, e.g., } \\
\text { sarcoidosis, Wegener's granulo- } \\
\text { matosis }\end{array}$ & $\begin{array}{l}\text { Wide spectrum of pachymeningeal and } \\
\text { leptomeningeal, often irregular enhancement, } \\
\text { typically affecting basilar meninges and the } \\
\text { cranial nerves }\end{array}$ & - \\
\hline
\end{tabular}

on contrast-enhanced CT or T1-weighted MR images as a thin, linear, and sometimes discontinuous enhancement, following the inner table of the skull, the falx, and the tentorium cerebelli. Typically, irritation and inflammation of the pachymeninges may arise from various benign or malignant processes, including transient postoperative changes, intracranial hypotension,

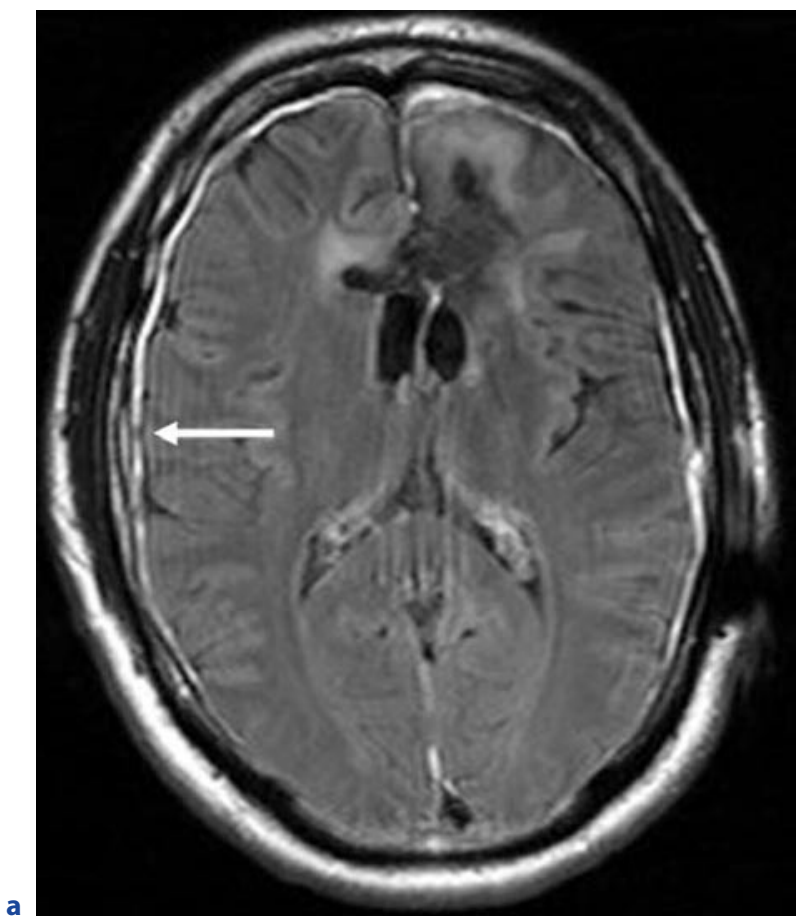

Fig. 11.1a,b. Intracranial hypotension. A 44-year-old patient who had been surgically treated for an oligodendroglioma of the left frontal lobe. Early post-operative MRI (3 T). a Axial FLAIR image. b Axial T1-weighted image after contrast administra- meningioma, metastases, lymphoma, and granulomatous disease that lead to vasocongestion and interstitial edema of the dura mater (Table 11.2). Classical imaging features of isolated inflammation of the pachymeninges include thickening and increased enhancement of the dura-arachnoid complex without enhancement of the sulci or brain surface (Fig. 11.1).

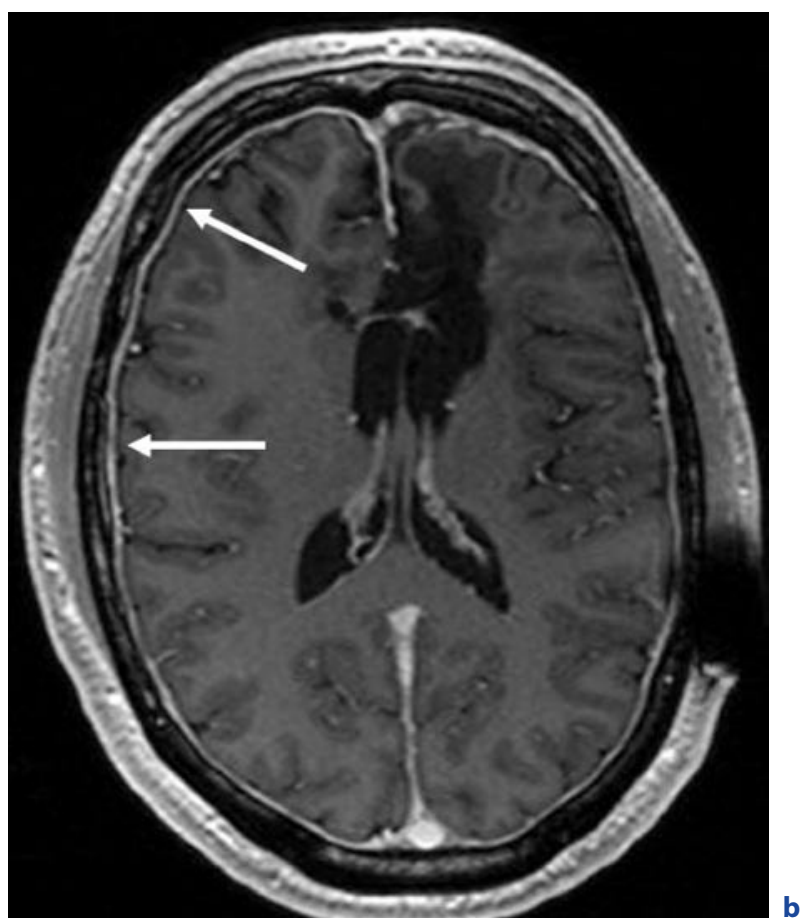

tion. Thickening and increased contrast enhancement of the pachymeninges with effusion into the subdural space (arrows in $\mathbf{a}, \mathbf{b}$ ), representing transient reactions of the dura-arachnoid complex due to intra-operative intracranial hypotension 


\section{3 \\ Bacterial Meningitis}

\subsection{1}

\section{Epidemiology, Clinical Presentation, Therapy}

Bacterial meningitis is the purulent infection of the cerebral and spinal leptomeninges. The incidence in the U.S. and Western Europe is about 5-10/100,000 persons. Meningitis is the most common form of CNS infection in children. Mortality ranges between 20 and $30 \%$, and neurological sequelae occur in $15-40 \%$ of surviving patients. Infection may arise from hematogenous spread during systemic infection, from a chronic suppurative focus, or per continuitatem during acute or chronic infections of the paranasal sinus, the middle or inner ear, or the mastoid. Another path of infection is brain injury with open or covered disruption of the dura mater, especially when the paranasal sinus or the air-filled cavities of the temporal bone are involved in the fracture.

The type of microorganism responsible for meningitis depends on the age of the patient and the way of infection: while in neonates the most common pathogens are group-B Streptococcus and Escherichia coli, in infants it is Haemophilus influenzae, Streptococcus pneumoniae, and Neisseria meningitidis. In adolescents and adults Streptococcus pneumoniae and Neisseria meningitidis are found most often. In a recent study on the epidemiology of 696 episodes of community-acquired meningitis in adults, $S$. pneumoniae was determined to be the responsible pathogen in $51 \%, N$. meningitides in $37 \%$, and Listeria monocytogenes in $4 \%$ of cases (VAN DE BEEK et al. 2004); however, due to vaccination against $H$. influenzae and S. pneumoniae, epidemiology of bacterial meningitis changes constantly.

In patients with ENT infections or after splenectomy, S. pneumoniae are the most common causative species in bacterial meningitis. After neurosurgery and in patients with open brain injury or with dural fistulae, S. aureus and Pseudomonas aeroginosa are found frequently beside $S$. pneumoniae and $H$. influenzae. Immunocompression and alcoholism are associated with Listeria monocytogenes, different types of Enterococcus, S. aureus, and S. pneumoniae.

In most of the cases meningitis starts with unspecific prodromes such as abnormal fatigue growing pains that may last for several hours or days before the onset of meningeal symptoms. On admission more than $90 \%$ of patients with bacterial meningitis present with at least two of the following four symptoms: fever; neck stiffness; changes in mental status - the "classical triad" - and headache. Headache is the most common symptom in meningitis and is found in more than $80 \%$ of the patients. Further symptoms indicating irritation of the leptomeninges are nausea, conjunctivitis, hyperesthesia, and hyperacusis. Approximately one fourth of the patients develop petechial rash, with $N$. meningitidis as the most frequent causative pathogen. One third of the patients have focal neurological deficits such as cranial nerve palsy, aphasia, hemiparesis, or seizures. These symptoms are associated with a lower level of consciousness and indicate a more severe course of the disease; they are more frequent in pneumococcal than in meningococcal meningitis (VAN DE BEEK et al. 2004). Bacterial meningitis may be complicated by cortical and subcortical infarctions due to septic vasculitis of the cerebral arteries, hydrocephalus, brain edema, empyema, or pyogenic abscess. Systemic complications result from bacteremia and comprise the Waterhouse-Friderichsen syndrome (e.g., meningococcal sepsis with disseminated intravasal coagulation and petechial bleeding), the adult respiratory distress syndrome (ARDS), and septic emboli into the lung or peripheral vessels.

Empirical antimicrobial treatment has to be started immediately after lumbar puncture and sampling blood cultures. Therapeutic regimens comprise antibiotic combinations from the penicillin, rifamycine, cephalosporin, and aminoglycoside groups. Antiedematous treatment with dexamethasone before or with the first dose of antimicrobial therapy has been proven to reduce the risk of unfavorable outcome, including mortality and neurological complications (WEISFELT et al. 2006). In cases of transmitted bacterial meningitis from infections of the sinus, mastoid, or middle ear, surgical removal of the focus is mandatory.

\subsection{2}

\section{Imaging}

In uncomplicated cases and the initial phase of the disease, neuroimaging might reveal normal findings of the brain and meninges; however, a CT exam should be performed in all patients with suspected bacterial meningitis prior to lumbar puncture to rule out brain edema and potential herniation. As a sign of beginning disturbance of the CSF circulation, CT might reveal mild enlargement of the ventricles and the SAS; the basal cisterns can be effaced. After administration of contrast medium, CT might demonstrate linear leptomeningeal enhancement in the sulci and cisterns that might increase during the course of the disease, especially if antibiotic treatment is not started or is started with de- 
lay (Kastrup et al. 2005). Parenchymal lesions are often difficult to distinguish; however, CT is a simple and fast tool for monitoring critically ill patients and to rule out severe complications of bacterial meningitis, such as resorptive hydrocephalus, swelling, venous thrombosis, or infarction. Besides cerebral structures, CT provides important information about probable pathologies of the skull base, the temporal bone, and the nasal sinus, such as fractures, purulent sinusitis, otitis media, or mastoiditis; therefore, CT of patients with bacterial meningitis should always include a reconstruction of the skull base in the "bone window".

Although not specific for a certain type of meningitis or a causative pathogen, contrast-enhanced T1weighted images have been proven to be more effective in demonstrating meningeal enhancement than contrast-enhanced CT (CHANG et al. 1990). Abnormal enhancement of the leptomeninges is usually asymmetrical and extends into the basal cisterns. Besides the importance of contrast-enhanced T1-weighted images, MRI may reveal an increased signal of the SAS on T2-weighted and FLAIR images, indicating leakage of the $\mathrm{BBB}$ with hypercellularity or increased proportions of proteins in the CSF (Fig. 11.2). When patients with bacterial meningitis develop focal neurological symptoms or seizures, MRI is the tool of choice to diagnose vascular or septic complications (Fig. 11.3). Diffusionweighted imaging is highly sensitive in depicting small cortical or deep white matter infarctions that are hardly seen with conventional MRI, whereas vascular changes can be visualized directly with MRA. In cases of severe stenosis, vasospasm, or vessel occlusion due to septic vasculitis, PerfMRI may help to get functional information about potential collaterals and the "tissue at risk," which is clinically helpful in deciding whether or not to use high-dose steroid therapy.

Subdural or epidural empyemas are slightly hyperintense relative to CSF and hypointense to white matter on T1-weighted images and hyperintense relative to CSF and white matter on T2-weighted images (Fig. 11.4). They are often associated with edema and mass effect. On DWI subdural empyema usually show high signal, whereas epidural empyema tend to be of low or mixed signal intensity (KASTRUP et al. 2005).
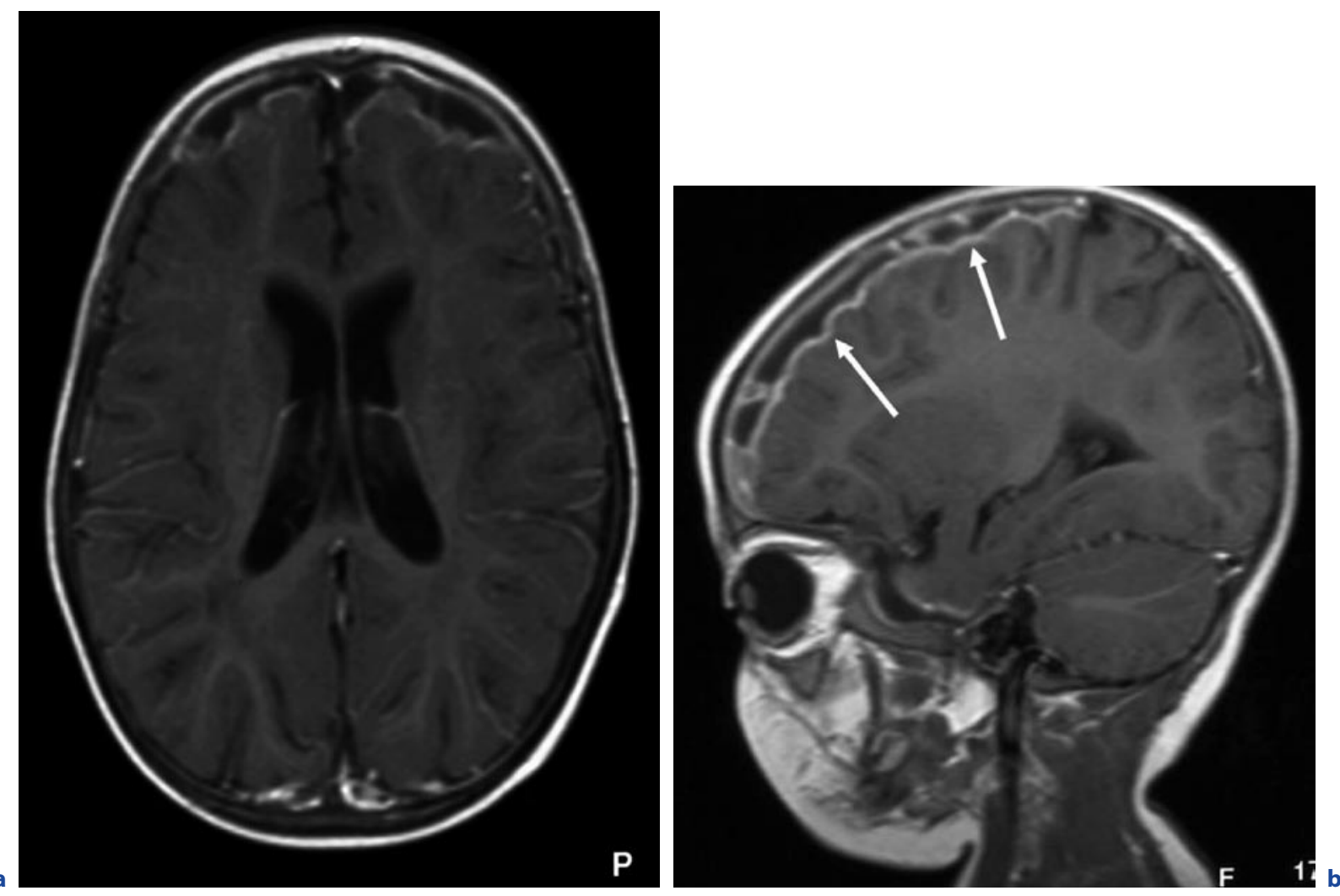

Fig. 11.2a-d. Pneumococcal meningitis in a 5-year-old child. a Axial T1-weighted image after contrast administration. b Sagittal T1-weighted image after contrast administration. c,d see next page 

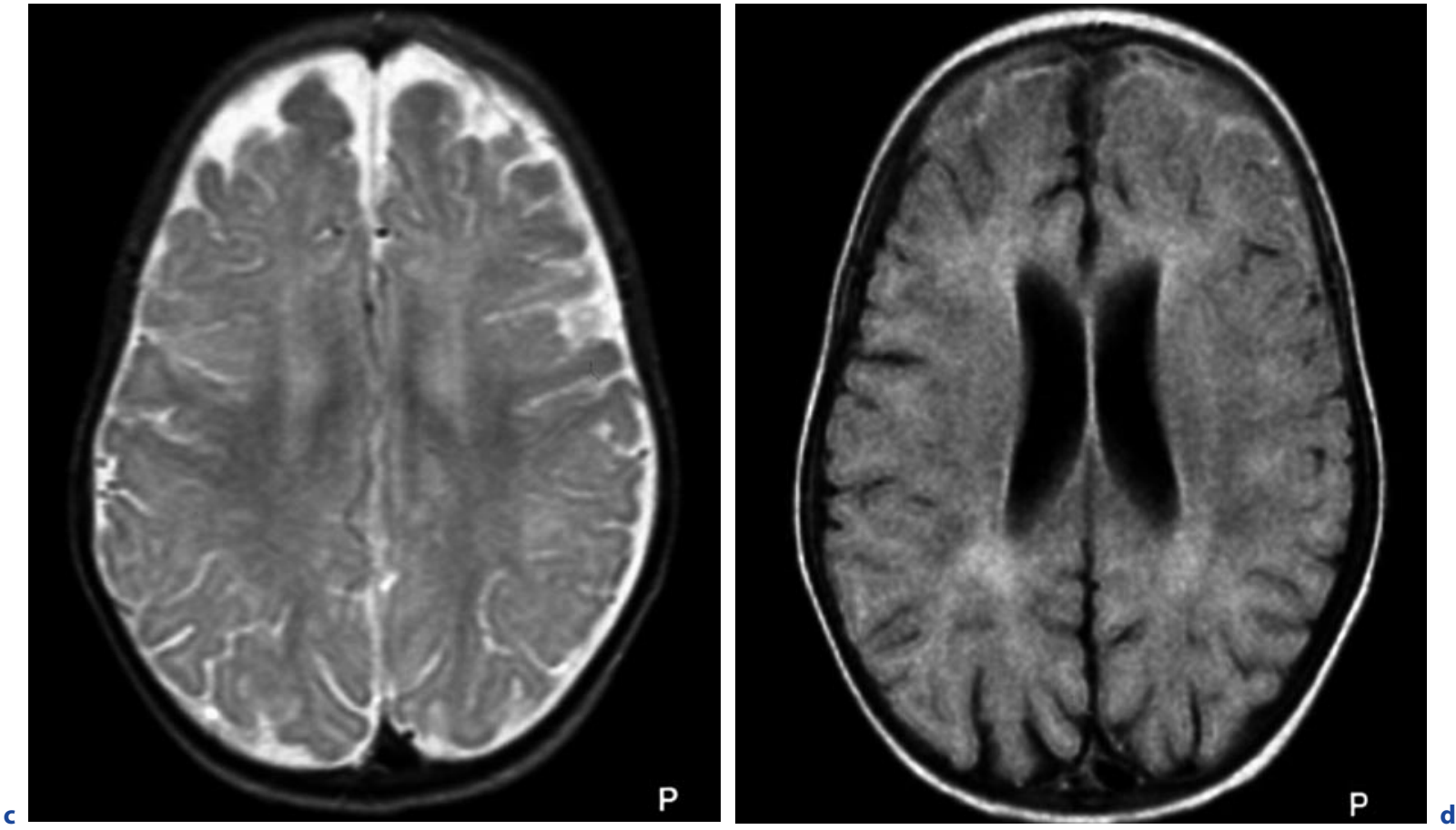

Fig. 11.2a-d. (continued) Pneumococcal meningitis in a 5-year-old child. c Axial T2-weighted image. d Axial FLAIR image. Bifrontal subdural exudates $(\mathbf{c}, \mathbf{d})$ with relatively high

signal compared with the CSF (d), indicating an elevated content of proteins 

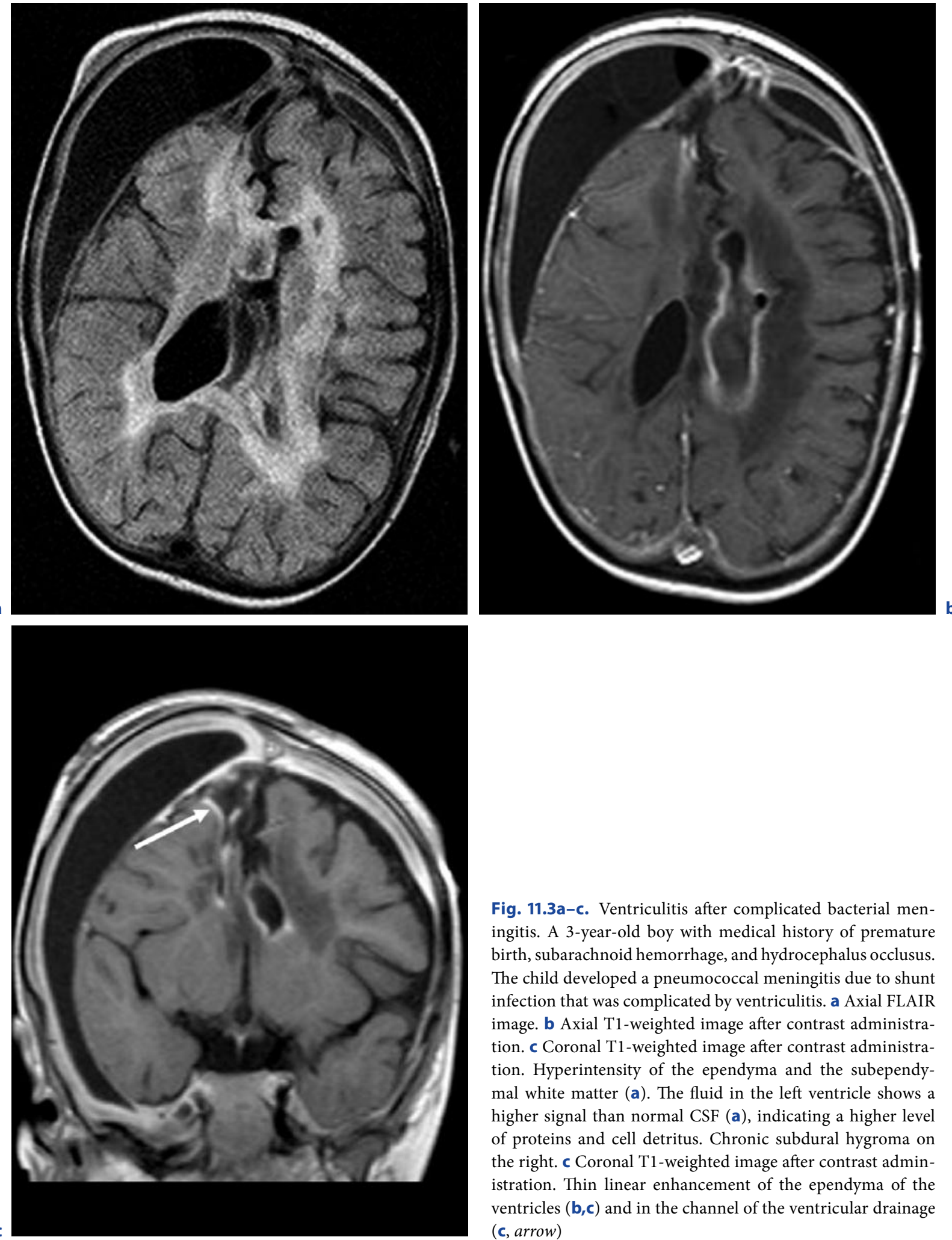

Fig. 11.3a-c. Ventriculitis after complicated bacterial meningitis. A 3-year-old boy with medical history of premature birth, subarachnoid hemorrhage, and hydrocephalus occlusus. The child developed a pneumococcal meningitis due to shunt infection that was complicated by ventriculitis. a Axial FLAIR image. b Axial T1-weighted image after contrast administration. c Coronal T1-weighted image after contrast administration. Hyperintensity of the ependyma and the subependymal white matter (a). The fluid in the left ventricle shows a higher signal than normal CSF (a), indicating a higher level of proteins and cell detritus. Chronic subdural hygroma on the right. c Coronal T1-weighted image after contrast administration. Thin linear enhancement of the ependyma of the ventricles $(\mathbf{b}, \mathbf{c})$ and in the channel of the ventricular drainage (c, arrow) 

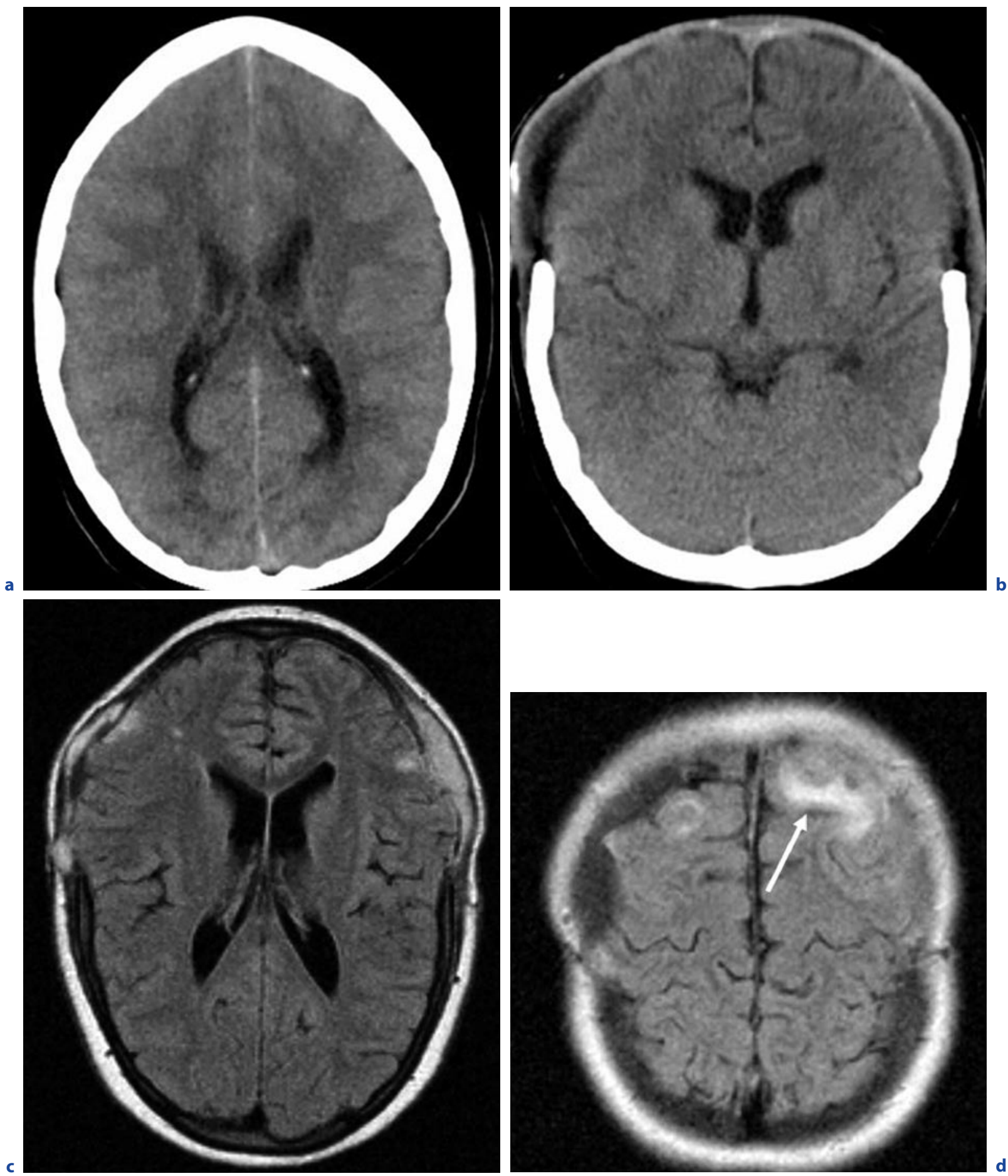

Fig. 11.4a-f. Pneumococcal meningitis with complicating epidural empyema. A 14-year-old girl that presented initially with clouded consciousness and recurrent generalized seizures due to meningococcal meningitis. Indication for bifrontal

hemicraniotomy was made due to pronounced general brain edema. a Axial CT before bifrontal hemicraniotomy. b Axial CT after bifrontal hemicraniotomy. $\mathbf{c}, \mathbf{d}$ Axial FLAIR images after bifrontal hemicraniotomy. e-f see next page 


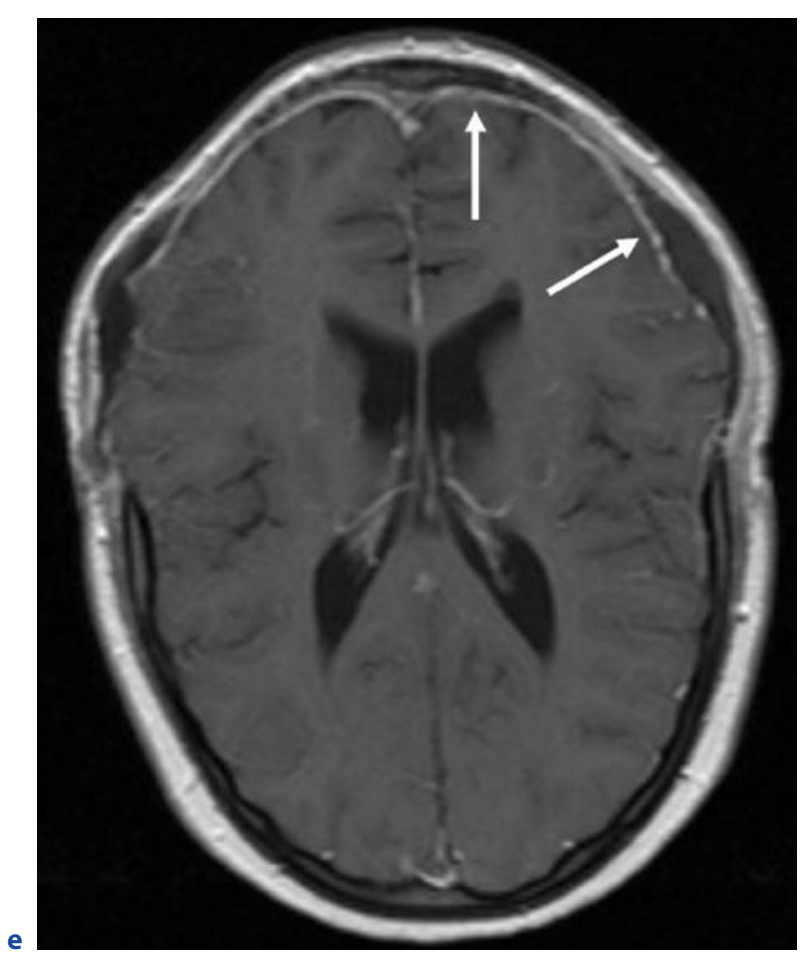

Fig. 11.4a-f. (continued) Pneumococcal meningitis with complicating epidural empyema. A 14-year-old girl that presented initially with clouded consciousness and recurrent generalized seizures due to meningococcal meningitis. Indication for bifrontal hemicraniotomy was made due to pronounced general brain edema. e Axial T1-weighted image after contrast administration after bifrontal hemicraniotomy. f Coronal T1weighted image after contrast administration after bifrontal hemicraniotomy. Axial FLAIR images $(\mathbf{c}, \mathbf{d})$ show hyperintense

\subsection{3}

\section{Differential Diagnosis}

Isolated enhancement of the leptomeninges is not specific for bacterial infection. Viral or fungal meningitis may present with similar changes of the meninges and the SAS, however, fungal meningitis normally causes a thicker, lumpy, or nodular enhancement in the SAS. Subarachnoid hemorrhage (SAH), acute stroke, high inspired oxygen, or imaging artifacts may cause an increased FLAIR signal in CSF that can be mistaken for infectious exudates in the SAS. Additionally, several primary and secondary tumors, e.g., medulloblastoma, breast cancer, or CNS lymphoma, may cause leptomeningeal enhancement as well, known as "carcinomatous meningitis." In the majority of cases, clinical presentation, medical history, and analysis of the CSF will provide the clue for differential diagnosis.

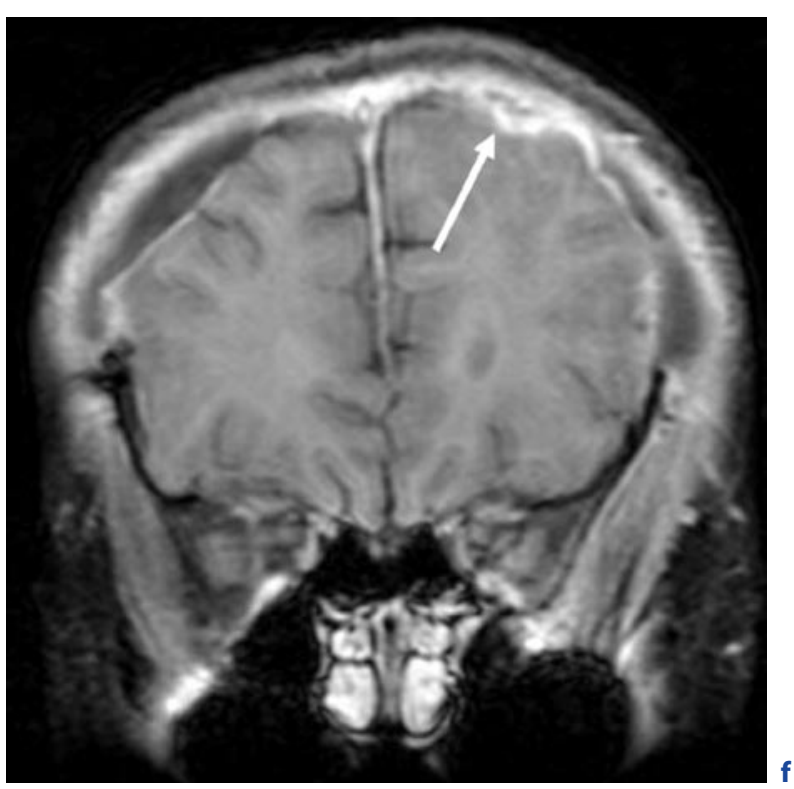

signal in the epidural fluid collection within the bifrontal craniotomy defects and hyperintense changes of the frontal cortex (arrow in d). One month after the surgical intervention, the patient developed seizures again. Axial and coronal contrastenhanced T1-weighted images $(\mathbf{e}, \mathbf{f})$ demonstrate bifrontal pachymeningeal enhancement, and additionally leptomeningeal and cortical enhancement of the adjacent frontal lobe (arrows), leading to the diagnosis of complicated bacterial meningitis with epidural empyema and beginning brain involvement

\section{4}

\section{Tuberculous Meningitis}

\subsection{1}

\section{Epidemiology, Clinical Presentation, Therapy}

In Central Europe tuberculous meningitis (TBM) has become relatively rare; however, due to socio-economic changes and the increasing number of HIV infections, the incidence of TBM is rising again. Worldwide, more than 8 million patients come down with TB annually. The TBM is the most frequent manifestation of CNS $\mathrm{TB}$ and is more common in children. The meninges are affected secondarily through hematogenous spread in patients with miliary tuberculosis or from ruptured tuberculous granulomata. In adults, the primary focus often cannot be identified. 
Clinically, TBM starts with unspecific symptoms such as fatigue, ill humor, diminished appetite, elevated temperature, and headache that may last for several weeks. Signs of meningeal affection often begin slowly and without disturbances of consciousness. Because of the predominant affection of the basal parts of the meninges, TBM frequently causes cranial nerve palsy, primarily of the abducent nerve, the oculomotoric nerve, and the facial nerve. In severe cases TBM may affect also caudal cranial nerves and spinal nerves. Hydrocephalus and secondary vasculitis are main complications. Mortality is still $10-20 \%$; it is even higher in underdeveloped countries and after delayed diagnosis.

Treatment has to be started as soon as possible, even without direct proof of the causative pathogenic agent, when clinical presentation and liquor results (e.g., moderate pleocytosis of 100-200 cells, strong positive lactate and protein, low glucose) suggest the diagnosis of TBM. Modern regimens comprise an antituberculostatic combination with Isoniazid, Rifampicin, Pyracinamid, or Ethambutol for at least 2 months or longer, when CSF is still positive for tuberculosis. In complicated cases intrathecal injection of streptomycin has been proposed as adjunctive treatment. Steroids should be given to prevent secondary vasculitis.

\subsection{2}

Imaging

As the prognosis of TBM depends on starting treatment early, and confirmatory tests take longer time (culture, PCR), imaging of TBM is crucial for immediate diagnosis. The typical imaging feature of TBM is enhancement of the basal cisterns and the corresponding leptomeninges caused by dense fibrinous exudate in the SAS (Fig. 11.5). Basal meningeal enhancement is found in approximately $60 \%$ of TBM cases. Interestingly, recent studies have demonstrated that tuberculous meningitis is not only seen on contrast-enhanced T1-weighted images, but also on non-enhanced T1-weighted MT images, showing the inflamed meninges as distinct basal periparenchymal hyperintensity (KAMRA et al. 2004). This phenomenon is reported to be missing in patients with meningitis of other etiology; thus, visibility of the meninges on non-enhanced T1-weighted MT images has to be considered highly suggestive of tuberculous meningitis. Yet, one has to keep in mind that formation of exudates requires a competent immune system in the host, as a consequence meningeal enhancement is often less pronounced or even missing in elderly patients and individuals with immune deficiency (SRIKANTH et al. 2007).

Further imaging findings in TBM comprise hydrocephalus malresorptivus in approximately $50-80 \%$ and infarcts due to secondary vasculitis in $30-40 \%$ of TBM cases. Both ischemic and hemorrhagic infarcts are diagnosed best with DWI and T2*-sequences on MRI; however, studies comparing CT and MRI have shown that advantages of MRI are minimal in terms of hydrocephalus and basal enhancement. Advantages of MRI are confirmed for patients with cranial nerve palsy: Imaging should always be performed in the axial and coronal plane using contrast-enhanced T1-weighted images with fat saturation to check for potential cranial nerve enhancement in the basal cisterns, the cavernous sinus, or the orbital cavities (see also Chap. 5).

\subsection{3 \\ Differential Diagnosis}

Other causes of infectious and carcinomatous meningitis have to be considered for differential diagnosis. While TBM causes predominantly basilar meningeal enhancement, bacterial meningitis affects more often the leptomeninges of the convexities. Furthermore, the combination of meningitis and parenchymal lesions suggests tuberculosis; however, some primary or metastatic tumors and some parasitic CNS infections (e.g., coccidiomycosis) may be indistinguishable from TBM.

\section{5 \\ Viral Meningitis}

\subsection{1}

\section{Epidemiology, Clinical Presentation, Therapy}

Isolated inflammation of the meninges due to virus infection is rare. Most viruses cause encephalitis or meningoencephalitis (described in detail in Chap. 9). Virus predominantly affecting leptomeningeal structures and the underlying cortex are HSV type 1, the most common causative agent for viral meningoencephalitis, and the arbovirus, that causes tick-borne meningoencephalitis (TBME). Additionally, parainfectious meningitis is a common finding in many systemic viral infections and after antiviral vaccination. Initial symptoms of viral meningitis are lassitude, fever, headache, and an altered sensorium. Epileptic seizures, aphasia, hemiparesis, clouded consciousness, or psy- 

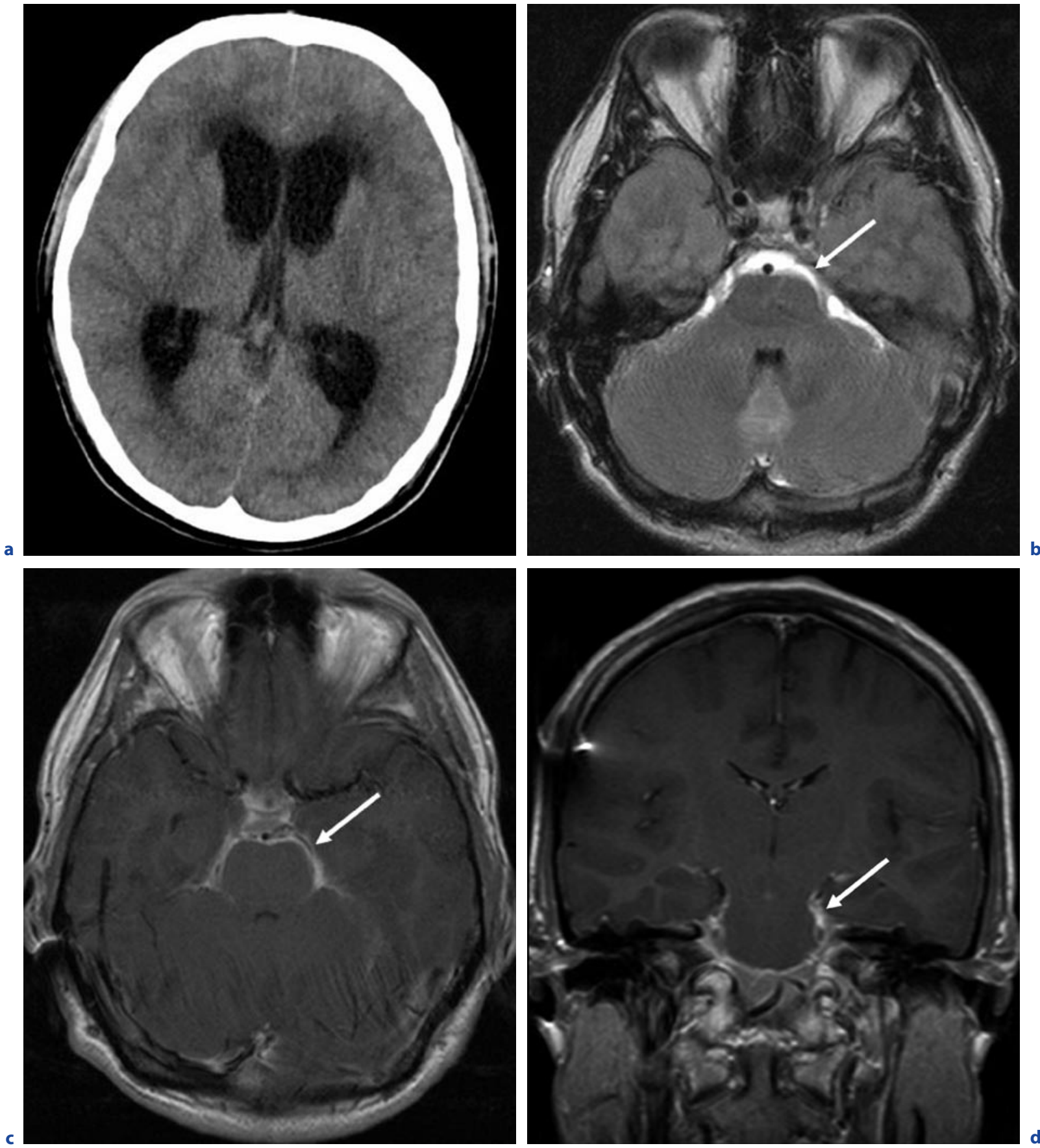

Fig. 11.5a-d. Tuberculous meningitis. A 24-year-old patient, with a history of intravenous drug abuse, presented with headache and abducent nerve palsy. a Axial CT. b Axial T2weighted image. c Axial T1-weighted image after contrast administration. d Coronal T1-weighted image after contrast administration. Initial CT (a) showed hydrocephalus. MRI

revealed basal meningitis with hyperintense exudates in the prepontine and basal cisterns and thick, linear leptomeningeal enhancement (arrow in $\mathbf{b}, \mathbf{c}$ ) around the pons, the pedunculi cerebelli, and within the quadrigeminal cistern. Examination of the CSF confirmed tuberculous meningitis 
chotic symptoms indicate an advanced "encephalitic state" of viral CNS infection. Analysis of the CSF might reveal mild, mononuclear pleocytosis $\left(<300 \mathrm{cells} / \mathrm{m}^{3}\right)$, normal glucose and lactate, and normal or elevated protein. Specific treatment with antiviral chemotherapeutics (e.g., acyclovir, gancyclovir, foscarnet) is not always possible and depends on the causative agent which should be diagnosed with PCR.
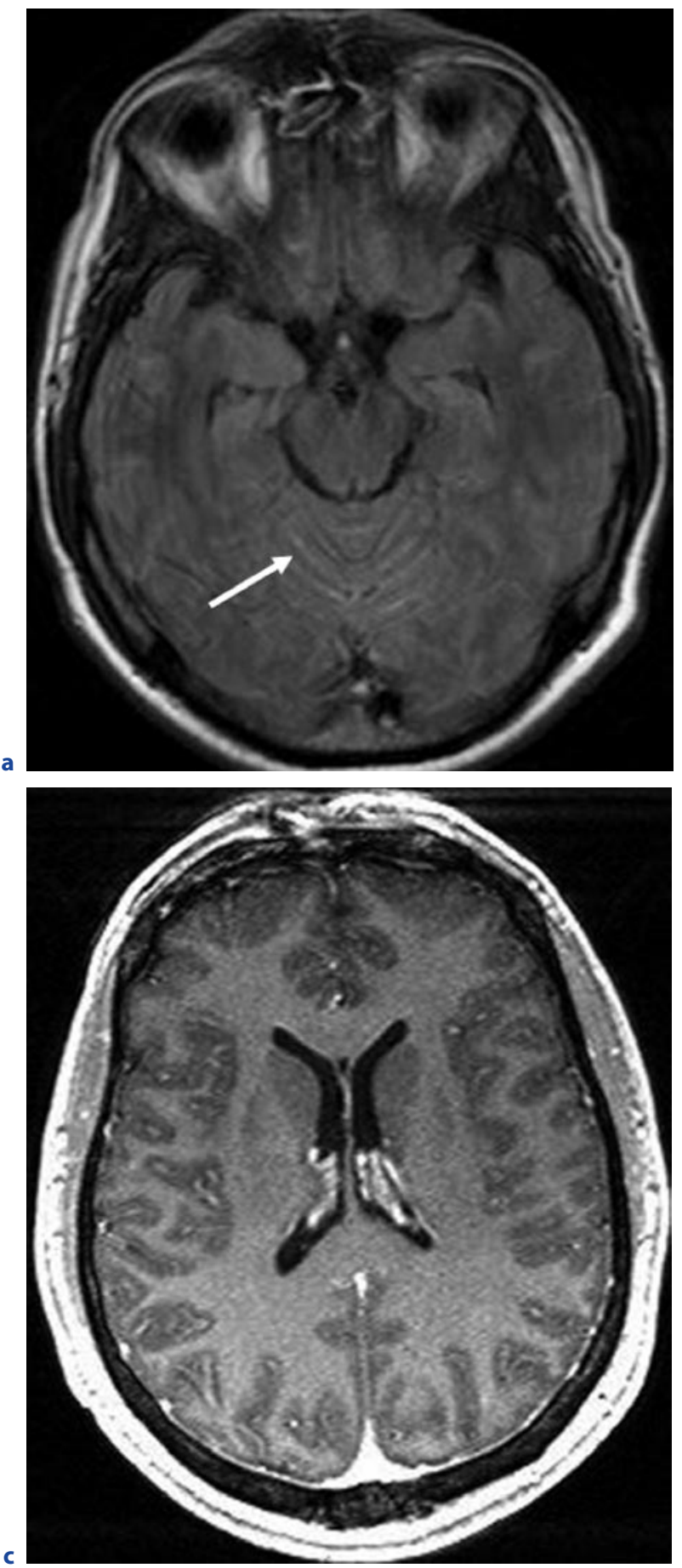

\subsection{2}

Imaging

In many cases and in early stages of viral meningitis, neuroimaging reveals no abnormalities or only unspecific changes such as focal or diffuse brain edema and swelling, or signal abnormalities in the SAS due to an increased protein content of the CSF (Fig. 11.6). In the

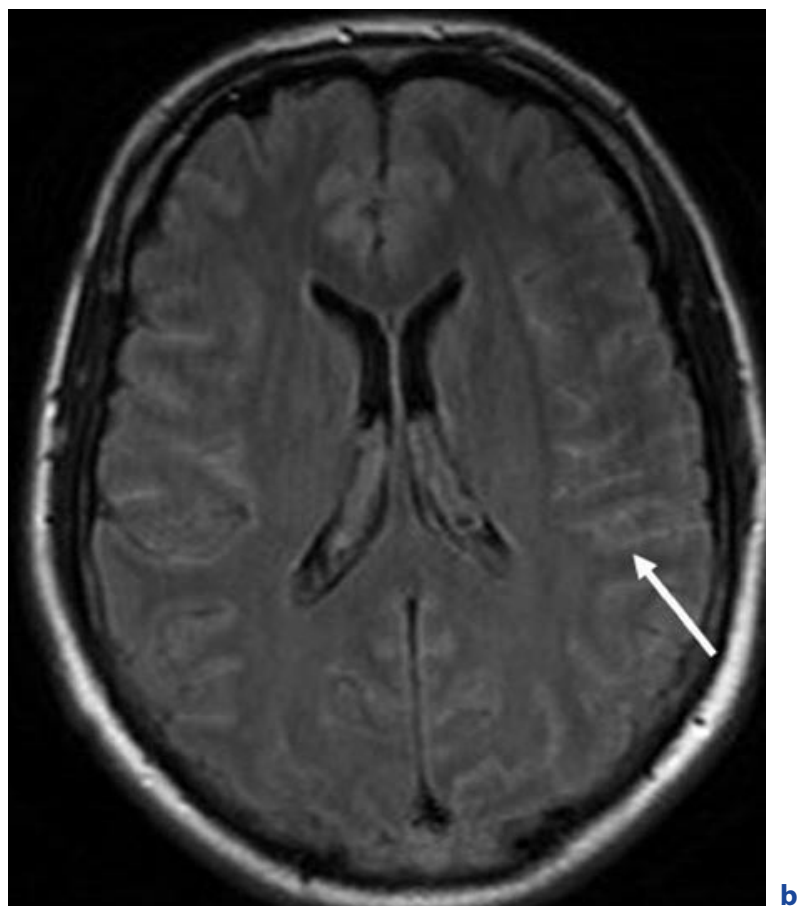

Fig. 11.6a-c. Viral meningitis. A 36-year-old patient, who was admitted with fever, headache, and confused mental state. Lumbar puncture revealed moderate pleocytosis, normal glucose, and lactate. a,b Axial FLAIR images. c Axial T1-weighted image after contrast administration. Slightly hyperintense signal in the subarachnoid space of the sulci (arrow in a,b), suggestive of protein-rich inflammatory exudates due to viral infection. Pathological enhancement of the meninges is absent (c) 
later stage of HSV-1-meningoencephalitis leptomeningeal and corticomedullary enhancement of the affected lobe may be visible; however, these changes follow the more specific changes of the disease with hyperintensity on T2-weighted and FLAIR images in limbic structures. Although patients with TBME develop meningitis in over $70 \%$, the majority of MRI scans are normal. Pathological findings indicating the presence of encephalitis comprise hyperintensities on T2-weighted images in the thalami, brain stem, caudate nucleus, or the cerebellum.

\subsection{3}

\section{Differential Diagnosis}

In cases of isolated viral meningitis, other infectious and neoplastic diseases, especially bacterial meningitis, have to be excluded. The diagnosis is made by testing of the CSF. In case of HSV meningoencephalitis, differential diagnosis includes paraneoplastic (limbic) encephalitis, ischemia, infiltrating neoplasm, other encephalitides (e.g., neurosyphilis), and status epilepticus.

\section{6}

\section{Fungal Meningitis}

\subsection{1}

\section{Epidemiology, Clinical Presentation, Therapy}

Fungal infections of the CNS are most frequent in immunocompromised patients with AIDS, long-lasting diabetes mellitus, or after organ or bone marrow transplantation. Fungal hyphae (e.g., Candida albicans) primarily affect the meningeal microcirculation, whereas infections with Cryptococcus neoformans, Aspergillus fumigatus, or Aspergillus flavus involve predominantly larger cerebral vessels, leading to encephalitis, brain abscess, or vasculitis. The most common fungal infection of the CNS is cryptococcal meningoencephalitis, followed by aspergillosis and candidiasis. Cryptococcal meningoencephalitis manifests weeks after asymptomatic lung infection with clinical symptoms of basal meningitis, cranial nerve palsy, and hydrocephalus. The diagnosis is made through direct confirmation of the fungus in the CSF. Even under intensified treatment with amphotericin B and 5-flucytosine, mortality of cryptococcal meningoencephalitis is high; treatment is successful in only $30-50 \%$ of cases.
Aspergillus may reach the CNS via hematogenous spread or through direct invasion from sinunasal aspergillosis. The infection manifests predominantly with solitary or multiple brain abscess or granulomata, less often with meningitis or ventriculitis. Mortality of CNS infection with Aspergillus is extremely high.

\subsection{2 \\ Imaging}

Cryptococcal meningoencephalitis typically manifests with diffuse meningeal enhancement and ventriculitis beside characteristic cystic, punctuate lesions in the basal ganglia due to cryptococcal invasion of the perivascular spaces, the "soap-bubble lesions" (KAStrup et al. 2005; HäHnel et al. 2005). Hydrocephalus is found more often in immunocompetent patients as arachnoid reactions with exudates in the basal cisterns often do not manifest in immunocompromised individuals.

Imaging features of Aspergillus meningeal infection comprise pachymeningeal enhancement nearby the affected nasal sinus or the orbit; typically, bony structures are involved in the inflammatory process. More than $50 \%$ of the patients develop hemorrhagic infarctions due to vasculitis of the perforating arteries of the basal ganglia. Parenchymal aspergillosis may appear as edematous and hemorrhagic lesions or solid, ring-like enhancing "tumoral" lesions with low signal intensity on T2-weighted images, which may be due to paramagnetic elements such as manganese and iron as well as blood-breakdown products. As laboratory findings of fungal infection do not always confirm the causative pathogen, knowledge of neuroradiological appearance of aspergillosis is helpful for early diagnosis (see also Chap. 9).

\subsection{3 \\ Differential Diagnosis}

Other infectious and neoplastic diseases, especially bacterial meningitis and TBM, have to be excluded. Fungal meningitis often causes a thicker and more lumpy meningeal enhancement than bacterial or viral meningitis. In case of pachymeningeal enhancement and involvement of the skull base, neoplasms arising from the nasopharynx of the sinus have to be ruled out. 


\section{7 \\ Neurosyphilis}

\subsection{1 \\ Epidemiology, Clinical Presentation, Therapy}

Syphilis is a curable sexually transmitted disease caused by the spirochete Treponema pallidum. The use of penicillin has reduced the incidence of syphilis during the past century; however, the trend has reversed since the 1980s due to the exchange of sex for drugs, unprotected sex, and the number of people with multiple sexual partners. The incidence of syphilis in industrialized countries is approximately $2-4 / 100,000$ persons. The central nervous system may be involved at any stage of the infection in about $5-10 \%$ of untreated patients; it is most common in patients with HIV infections. Neurosyphilis (neurolues) is classified into four syndromes: syphilitic meningitis; meningovascular syphilis; as well as parenchymatous and gummatous neurosyphilis, these latter ones ending in general paresis and tabes dorsalis.

Syphilitic meningitis occurs usually in the second stage of the disease within the first 2 years after infection. It is thought to be the consequence of direct meningeal inflammation due to small-vessel arteriitis. Patients present with headache, meningeal irritation, and cranial nerve involvement, especially the optic nerve, facial nerve, and the vestibulocochlear nerve. Meningovascular syphilis is assigned to the tertiary or late stage of syphilis. After prodromal symptoms that may last for weeks to months, focal deficits with unilateral numbness, paresthesias, hemiparesis, headache, vertigo, or psychiatric abnormalities are identifiable. These symptoms are caused directly by the inflammation of the meninges and the parenchyma or secondarily by infarctions and (aneurysm associated) bleedings due to vasculitis. The first-choice treatment for all manifestations of syphilis, including neurolues, is penicillin.

\subsection{2}

Imaging

There is only little systematic data about neuroradiological findings in neurosyphilis. Approximately one third of the patients have normal findings (BRIGHTBILL et al. 1995). The majority of cases presents with non-specific white matter lesions or cerebral infarctions, less often with cerebral gummata. Syphilitic meningitis is a rare finding: The leptomeninges may present with thick and lumpy enhancement; predominantly the basal cisterns and the prepontine SAS are affected. In case of cranial nerve palsy, the involved nerve shows swelling and increased enhancement. Vasculitis is a common finding in MRA or DSA. Typical features include stenosis and interruption of large and medium-large arteries, and vasculitic aneurysms (see also Chap. 4).

\subsection{3 \\ Differential Diagnosis}

In case of isolated syphilitic meningitis, other granulomatous diseases, especially tuberculosis, have to be excluded. The diagnosis is made by serological testing of the CSF and blood.

\section{8 \\ Neurosarcoidosis}

\subsection{1 \\ Epidemiology, Clinical Presentation, Therapy}

Sarcoidosis is a multisystem inflammatory disease characterized by non-caseating epitheloid-cell granulomas. Women are affected more frequently than men, and the onset of the disease is in the third and fourth decade. The CNS is involved in approximately $10 \%$ of cases. In up to $50 \%$ of cases, neurosarcoidosis is asymptomatic or self-limiting, and the remainder develop a chronic relapsing-remitting course of the disease. Typically, patients present with cranial nerve palsy, most often the facial nerve or the abducence nerve is affected. Further symptoms comprise meningeal irritation, signs of increased intracranial pressure, seizures, and hypothalamic or pituary gland dysfunction (e.g., diabetes insipidus). A specific treatment is not known, but corticosteroids are useful in most patients.

\subsection{2}

Imaging

Plain radiography and native CT may reveal osteolytic skull lesions. Contrast-enhanced CT and MRI will show a wide spectrum of leptomeningeal enhancement, especially of the basal SAS, and cranial nerve involvement; therefore, imaging of neurosarcoidosis should always 
include contrast-enhanced T1-weighted images with a slice thickness of 1-2 mm. Typically, neurosarcoid infiltration affects the infundibulum, chiasma, and hypothalamus. The granulomatous changes of the pachymeninges and in the SAS show focal or diffuse low signal intensity on T2-weighted images and isointense on T1weighted images. More than $50 \%$ of the patients present with periventricular hyperintense lesions on FLAIR and T2-weightes images, representing perivascular infiltrates in the Virchow-Robin spaces and vasogenic edema due to vasculitis of the small arteries. Diffusionweighted imaging helps to distinguish acute cytotoxic edema from neurosarcoid-induced vasogenic edema (see also Chaps. 2 and 12).

\subsection{3}

\section{Differential Diagnosis}

Known as the "great mimicker," differential diagnosis of neurosarcoidosis should include all types of meningitis, as well as neoplastic infiltration of the dura or the meninges (e.g., meningioma). The diagnosis is made by analysis of the chest X-ray, laboratory findings, and the CSF. In unclear cases, biopsy has to be considered for validation of the diagnosis.

\section{References}

Brightbill TC, Ihmeidan IH, Post MJD, Berger JR, Katz DA (1995) Neurosyphillis in HIV-positive and HIV-negative patients: neuroimaging findings. Am J Neuroradiol 16:703-711

Chang KH, Han MH, Roh JK, Kim IO, Han MC, Kim CW (1990) Gd-DTPA-enhanced MR imaging of the brain in patients with meningitis: comparison with CT. Am J Neuroradiol 11(1):69-76

Hähnel S, Storch-Hagenlocher B, Kress B, Stippich C, Sartor K, Forsting M, Seitz A, Jansen O (2005) Infectious diseases of brain parenchyma in adults: imaging and differential diagnosis aspects. Rofo 177(10):1349-1365

Kamra P, Azad R, Prasad KN, Jha S, Pradhan S, Gupta RK (2004) Infectious meningitis: prospective evaluation with magnetization transfer MRI. Br J Radiol 77(917):387-394

Kastrup O, Wanke I, Maschke M (2005) Neuroimaging of infections. NeuroRx 2(2):324-332

Smirniotopoulos JG, Murphy FM, Rushing EJ, Rees JH, Schroeder JW (2007) Patterns of contrast enhancement in the brain and meninges. Radiographics 27(2):525-551

Srikanth SG, Taly AB, Nagarajan K, Jayakumar PN, Patil S (2007) Clinicoradiological features of tuberculous meningitis in patients over 50 years of age. J Neurol Neurosurg Psychiatry 78(5):536-538

Van de Beek D, de Gans J, Spanjaard L, Weisfelt M, Reitsma JB, Vermeulen M (2004) Clinical features and prognostic factors in adults with bacterial meningitis. N Engl J Med 351(18):1849-1859

Weisfelt M, Hoogman M, van de Beek D, de Gans J, Dreschler WA, Schmand BA (2006) Dexamethasone and long-term outcome in adults with bacterial meningitis. Ann Neurol 60(4):456-468 